\title{
Examining the effects of a mindfulness-based professional training module on mindfulness, perceived stress, self-compassion and self-determination
}

\author{
S Whitesman, ${ }^{1} \mathrm{MB}$ ChB; $\mathbf{R}$ Mash, ${ }^{2} \mathrm{MB}$ ChB, DRCOG, DCH, MRCGP, FCFP, PhD \\ ${ }^{1}$ Division of Family Medicine and Primary Care, Faculty of Medicine and Health Sciences, Stellenbosch University and Institute for Mindfulness South Africa, Cape Town, South Africa \\ ${ }^{2}$ Division of Family Medicine and Primary Care, Faculty of Medicine and Health Sciences, Stellenbosch University, Cape Town, South Africa
}

Corresponding author: S Whitesman (simonw@lantic.net)

Background. Mindfulness-based interventions (MBIs) have been shown to be effective in a wide range of health-related problems. Teaching and research with regard to MBIs have largely been conducted in the USA and Europe. The development of teachers of MBIs requires that they embody the practice of mindfulness and acquire pedagogical competencies. Stellenbosch University and the Institute for Mindfulness South Africa have launched a new and innovative training programme consisting of 4 modules, with a blend of residential retreats and e-learning. Internationally, this is the first study that specifically investigates the effects of mindfulness on the mental state of health professionals being trained to teach MBIs in their clinical practice.

Objectives. To evaluate the first 9-week module in terms of its effect on mindfulness practice, self-determination, self-compassion and perception of stress. Methods. This is a before-and-after study of 23 participants, using 4 validated tools: Kentucky Inventory of Mindfulness Skills, Self-Determination Scale, Self-Compassion Scale, Perceived Stress Scale.

Results. There were significantly increased scores $(p<0.05)$ for all 4 aspects of mindfulness practice (observing, describing, acting with awareness and accepting without judgement) and self-compassion. There was also a significant decrease in the perception of stress, but no effect on self-determination scores, which were already high at baseline.

Conclusion. Potential teachers of MBIs in South Africa demonstrated significant gains in their own mindfulness practice and self-compassion as well as decreased perception of stress during the first module of the training programme. Further research will follow as this group completes the entire programme. Afr J Health Professions Educ 2015;7(2):220-223. DOI:10.7196/AJHPE.460

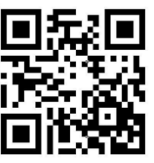

Mindfulness is moment-to-moment awareness and can be intentionally cultivated by paying attention in a specific way, i.e. in the present moment, non-judgementally, nonreactively and open-heartedly. ${ }^{[1]}$ While mindfulness is a core element of Buddhist philosophy, early Western psychologists, such as William James, recognised the relevance of mindfulness, although it was not until the 1980s that the academic literature began to earnestly grapple with and fully understand its construct and application. ${ }^{[2]}$

More generally, high mindfulness scores have been shown to predict selfregulated behaviour, positive emotional states and improved psychological wellbeing. ${ }^{[3]}$ High levels of mindfulness have also been shown to correlate inversely with dissociation, alexithymia, and general psychological distress. ${ }^{[4]}$

Mindfulness-based interventions (MBIs) have been developed to reduce symptoms across a wide range of populations and disorders, such as anxiety and depression, chronic pain, irritable bowel syndrome, HIV/AIDS and eating disorders. ${ }^{[5]}$ Research shows that mindfulness-based approaches induce neuroplastic changes in the brain, especially the frontal-limbic axis, ${ }^{[6]}$ enhance humoral immunity, ${ }^{[7]}$ and may reduce relapse in patients who abuse substances. ${ }^{[8]}$

MBIs therefore have a therapeutic place in healthcare services, and healthcare professionals such as psychologists or family physicians have shown interest in learning how to teach MBIs to their patients. To deliver MBIs to patients, healthcare professionals have to acquire knowledge of the approach and be able to practise mindfulness. The training of professionals to deliver MBIs rests on a set of core competencies that are introduced and assessed over the course of the programme. These competencies include guiding formal mindfulness practices, facilitating group processes and having good relational skills, linking one's direct experience of mindfulness with course themes and embodying the qualities of mindfulness through modelling a particular way of being. ${ }^{[9]}$

The secularisation of mindfulness in the form of MBIs and its strong research foundation has led to its growing popularisation. There is a risk that the mainstreaming of mindfulness may lead to a slow dilution in the integrity of the approach. In this context, the effective and ethical training of teachers to deliver MBIs in diverse contexts is an important development in the pedagogy of mindfulness. In South Africa (SA) it is the motivating principle behind the training programme being offered by the Faculty of Medicine and Health Sciences at Stellenbosch University in collaboration with the Institute for Mindfulness SA, a nonprofit organisation committed to the training, research and application of MBIs. The Certification in Mindfulness-Based Interventions is a 60-credit training, which provides participants with experiential and theoretical exposure to mindfulness and mindfulness-based approaches in a supportive learning environment, with a view to professional application within existing spheres of expertise.

The training consists of 4 modules structured as a series of 8 - 10 week courses. Methods of learning combine residential training retreats and distance learning strategies, including online supervision and discussion, self-directed mindfulness practice with formal techniques and integration of mindfulness into daily life, practising and exploring teaching skills 
(such as guiding practices and inquiry), self-reflective journaling, reading and critiquing peer-reviewed literature and writing essays for assessment purposes.

The 4 modular themes are as follows:

Module 1. Mindfulness from the inside-out: An introduction to mindfulness and mindfulness-based approaches. The objective of this module is for participants to begin to explore the practice of mindfulness experientially and understand the theoretical basis and clinical context of its application.

Module 2. Blending form and essence: Exploring the key elements of a mindfulness-based intervention. The intent of this module is to understand the architecture, essential content and process of an MBI.

Module 3. The ground beneath our feet: The foundations of mindfulness. The aim of module 3 is for participants to experience the depth of mindfulness practice and the ethos from which it arose, to encounter the framework for exploring suffering and its transformation, and to consider the relationship between the source of these teachings and their contemporary expression.

Module 4. Mindfulness at work: Refining the practice, refining the teaching, and the space between. The purpose behind the final module is to translate the theory into practice and to consider how to develop a personal intention and capacity for teaching through intensive exploration of guiding mindfulness practices in a variety of contexts under peer supervision.

Most of the formal training programmes in mindfulness-based approaches take place in the USA and Europe, up to and including Master's level degrees. Our training programme is the first of its kind in SA and research into its uptake, effectiveness, applicability and impact on participants and the communities they serve is essential.

While MBIs are increasingly viewed as structured stepwise approaches to various health-related issues, the ethical debate among those offering university-based training programmes focuses on the centrality of the teachers' embodiment of the qualities of mindfulness itself (such as presence, centredness, compassion) and the effect of the subjective state of the teacher on the communication and uptake of the curricular elements by the participants. ${ }^{[9]}$ In this context, monitoring and evaluating the inner states of those undergoing teaching training development - and continuing this evaluation process after completion of the training - is potentially an important addition to the growing literature on the pedagogy of mindfulness.

The essential quality of mindfulness is a combination of heightened attentional capacity and compassion. The latter is the ability to be receptive to, understanding of and responsive to our suffering and to that of others. In this context, there can be no compassion separate from self-compassion. Furthermore, compassion encompasses a softening of the habitual tendency to judge the contents of the present moment in a manner that reduces engagement with the actuality of what is occurring. This attitude of lessening the negating quality of mind is not a loss of discernment. Rather, the increase in mindfulness leads to a more refined and accurate perception of what is actually happening to and in an individual in successive moments of awareness, and the capacity to discern reality from reactivity. One of the consequences is to deal with stressful experiences on their own terms rather than from the reflexive overlay of conditioning. Such mindfulness becomes a valuable means to reduce the impact of psychological stress - much of which arises from a distorted perception of the present reality - at the same time embedding the capacity to respond rather than react to stressors within an individual's awareness. This capacity to choose a response to stressors, rather than simply reacting, is referred to as self-determination. Effective mindfulness practice, therefore, should lead to enhanced self-compassion and selfdetermination and a reduced perception of stress.

There are no studies in the literature that describe changes in levels of mindfulness and other mental capacities (e.g. compassion) as a result of such a professional training programme. The aim of this study was to evaluate the first module of the new short course on mindfulness practice at Stellenbosch University. The study evaluated changes in mindfulness practice, self-determination, self-compassion and perception of stress among participants.

\section{Methods}

\section{Study design}

We evaluated a short course on mindfulness, using before-and-after quantitative measures of mindfulness, self-determination, self-compassion and perceived stress.

\section{Setting}

Healthcare professionals participated in a 9-week short course on mindfulness practice at Stellenbosch University in collaboration with the Institute for Mindfulness SA. The short course was the first module in a larger programme on MBIs in healthcare that will follow. It consisted of an initial 1-week residential retreat, which intensively focused on the practice of mindfulness. The first day started at the Faculty of Medicine and Health Sciences, Stellenbosch University, with training in the computer laboratory and an overview of the course. The retreat was followed by 8 weeks of further theory, practice during normal daily living and reflection delivered by an internet-based program. The course was designed for healthcare practitioners, who were assessed on their contributions to online discussions and 2 final written assignments. One of the assignments was a personal narrative on their experience and exploration of mindfulness practice over the previous 8 weeks, and the second was a critique of the theory of mindfulness. The cohort consisted predominantly of medical practitioners and psychologists in clinical practice in private and state sectors. Participants enrolled in the training programme with the intention of learning how to teach mindfulness to patients in a secular context through deepening their personal practice while exploring the pedagogical aspects of its application.

\section{Study population}

The 23 students registered for the course were invited to participate in the study.

\section{Data collection}

Data were collected at the start of the course (at the introduction on day 1) and immediately after the end of the course as part of the feedback. The Kentucky Inventory of Mindfulness Skills is a validated 39-item self-report tool that was used to measure mindfulness on 4 scales: observing, describing, acting with awareness, and accepting without judgement. ${ }^{[10]}$ The SelfDetermination Scale, a 10-item tool, assessed differences in people's selfawareness and the extent to which they feel a sense of choice with regard to their behaviour. ${ }^{[1]}$ The Self-Compassion Scale is a validated 26-item questionnaire that was used to rate how people responded to themselves in difficult times. ${ }^{[12]}$ The Perceived Stress Scale is a validated 10-item questionnaire employed to measure perceived stress over the last month. ${ }^{[13]}$ 


\section{Data analysis}

Quantitative data from the questionnaires were entered, checked and cleaned in a Microsoft Excel spreadsheet and analysed (in Statistica) with the assistance of the Centre for Statistical Consultation. Before-and-after numerical data from paired groups were compared with a nonparametric Wilcoxon-signed rank test.

\section{Ethical considerations}

The study was approved by the Health Research Ethics Committee, Stellenbosch University (N13/07/100).

\section{Results}

The study population comprised 18 women and 5 men with a mean age of 44.2 (range 32 76) years. The group included 5 psychologists, 5 medical practitioners, 4 counsellors or coaches, 3 nurses, 3 professionals involved in leadership development, 1 social worker, 1 physiotherapist and 1 volunteer working with student groups. Eighteen participants had some previous exposure to mindfulness practice, while 5 had no prior exposure. Eighteen were self-employed or working in the private sector, 4 worked in the public sector and 1 was doing voluntary work.
Table 1 presents the results of the questionnaires and shows a statistically significant improvement in the practice of mindfulness. Participants showed a significant improvement in all 4 key dimensions of mindfulness: the ability to observe or direct one's attention to internal and external phenomena; ability to non-judgementally describe thoughts and feelings; ability to act with full awareness of what one is doing in the present moment; and ability to accept or allow what is happening without judging the experience. Participants improved despite being a self-selected group, most of whom had some previous exposure to mindfulness practice.

Participants also improved significantly in all the dimensions of self-compassion, which measure how someone acts towards him/herself while experiencing difficult circumstances: selfkindness, avoiding self-judgement, identification with others, avoiding isolation, practice of mindfulness, and avoiding over-identification with difficulties.

Participants reported improved mindfulness and self-compassion and a significant decrease in their perception of stress during the previous month.

There was no change in the extent to which participants reported functioning in a self-
Table 1. Results of the questionnaires at baseline and follow-up (N=23)

\begin{tabular}{|c|c|c|c|}
\hline Item & $\begin{array}{l}\text { Baseline mean } \\
(95 \% \mathrm{CI})\end{array}$ & $\begin{array}{l}\text { Follow-up mean } \\
(95 \% \mathrm{CI})\end{array}$ & $p$-value \\
\hline \multicolumn{4}{|c|}{ Kentucky Inventory of Mindfulness Skills (summative score) } \\
\hline Observe $(12-60)$ & $40.9(37.6-44.2)$ & $47.1(44.7-49.5)$ & $<0.001$ \\
\hline Describe $(8-40)$ & $28.4(25.5-31.3)$ & $30.3(28.1-32.6)$ & 0.018 \\
\hline Acting with awareness $(10-50)$ & $31.4(28.5-34.3)$ & $34.9(32.2-37.5)$ & 0.001 \\
\hline Accept without judgement $(9-45)$ & $32.7(29.5-35.9)$ & $35.5(33.0-37.9)$ & 0.016 \\
\hline \multicolumn{4}{|c|}{ Perceived Stress Scale (summative score) } \\
\hline Perceived Stress Scale $(0-40)$ & $16.5(13.5-19.4)$ & $12.8(9.8-15.7)$ & 0.012 \\
\hline \multicolumn{4}{|l|}{ Self-Compassion Scale (mean score) } \\
\hline Humanity $(1-5)$ & $3.3(3.0-3.7)$ & $3.8(3.5-4.1)$ & 0.005 \\
\hline Isolation $(1-5)$ & $3.4(3.0-3.8)$ & $3.8(3.4-4.1)$ & 0.032 \\
\hline Judgement $(1-5)$ & $3.2(2.8-3.6)$ & $3.6(3.3-4.0)$ & 0.012 \\
\hline Kindness $(1-5)$ & $3.3(2.9-3.7)$ & $3.8(3.4-4.1)$ & 0.002 \\
\hline Mindful (1 - 5) & $3.4(3.0-3.9)$ & $3.8(3.5-4.1)$ & 0.051 \\
\hline Over-identified (1 - 5) & $3.1(2.8-3.5)$ & $3.4(3.1-3.8)$ & 0.047 \\
\hline Total $(1-5)$ & $3.3(3.0-3.6)$ & $3.7(3.4-3.9)$ & 0.002 \\
\hline \multicolumn{4}{|c|}{ Self-Determination Scale (mean score) } \\
\hline Aware (1 - 5) & $4.0(3.7-4.3)$ & $4.0(3.8-4.3)$ & 0.681 \\
\hline Choice (1 - 5) & $3.8(3.5-4.2)$ & $4.0(3.8-4.3)$ & 0.117 \\
\hline Total $(1-5)$ & $3.9(3.7-4.2)$ & $4.0(3.8-4.2)$ & 0.346 \\
\hline
\end{tabular}

determined way. This especially relates to a sense of choice and control over one's behaviour. The reported self-determination was relatively high at baseline and did not change significantly during the course.

\section{Discussion}

Internationally, this is the first study that specifically investigates the effects of mindfulness on the mental state of those being trained to become teachers of mindfulness-based approaches. The results suggest that the initial stages of the abovementioned training at Stellenbosch University increased levels of self-compassion and mindfulness, which were accompanied by a decrease in the perception of stress.

There is an emerging literature on MBI teacher training that focuses on pedagogical elements. This literature speaks in depth to the core competencies required and offers a framework in which professionals training to teach MBIs are assessed. Assessment focuses on skills development and capacity to communicate the key elements of a curriculum effectively, skillfully, compassionately and in an embodied way. ${ }^{[14]}$ The initial data in this study suggest that compassion, one of the core elements of mindfulness, is enhanced with this training methodology and serves to support one of the key aspects required in teacher development. ${ }^{[14]}$

The complex and multi-levelled challenges in our society demand innovative approaches to integrating mindfulness, without compromising the standards of training, leading to benefit in the therapeutic domain. The course offered at Stellenbosch University follows the international trend of combining residential and distance learning elements, although the curriculum structure is unique to this programme, considering cost, context and accessibility to make the training available to healthcare professionals in other African countries.

There are a number of potential benefits of training professionals in mindfulness-based approaches. Mindfulness and compassion are universal capacities and may potentially be used in diverse contexts. MBIs can be delivered in group format at primary care level and at relatively low cost (one only needs a room and teacher fees), is highly participatory and empowering for participants, and the skills acquired may subsequently be developed beyond the conclusion of a programme at no cost. Furthermore, MBIs are highly adaptable in terms of structure, duration and language without losing the essential elements or thread of the curriculum. Research 
on the implementation of MBIs in the inner cities in the USA supports this adaptability. ${ }^{[15]}$ However, similar contextual adaptations in Africa remain to be explored.

The lack of a control group, and participants in the training programme having been self-selected, limits the generalisability of these findings. It is possible that unmeasured confounding factors influenced the results, although none were identified. It is also possible that obsequiousness bias could have influenced the responses of the participants to the questionnaires, although the follow-up questionnaire was completed online and not in the presence of the tutor. Moreover, this is the first of 4 modules; at this stage conclusions cannot be drawn with regard to the effects on the overall training programme.

Ongoing research might consider whether the inner state of the teacher correlates with the therapeutic outcome in MBIs. There is evidence for this in other contexts, where increased levels of empathy among primary care physicians correlated with a reduction in diabetic complications. ${ }^{[16]}$

High workload, low numbers of healthcare workers, poor infrastructure and an unsupportive organisational culture all contribute to high levels of stress, burnout and depression. ${ }^{[17]}$ Quality of care and the ability to care for others diminish with burnout and lower levels of resilience among healthcare professionals. The changes seen in this study in levels of mindfulness, self-compassion and stress may not only help to better prepare healthcare professionals as teachers of MBI but also have a positive impact on their clinical engagement and quality of care due to enhanced wellbeing and resilience. ${ }^{[18]}$ In the SA context, ongoing research into the impact of healthcare professional training programmes, based on the practice of mindfulness, should be explored in educational and clinical settings.

\section{Conclusion}

The first module of a training programme in MBIs led to significant improvements in mindfulness practice and levels of stress and self- compassion among participating healthcare professionals. There was no change in the degree of self-determination. Such changes may assist healthcare professionals to become teachers of mindfulness practice and be more resilient in the clinical workplace. Further research is needed to evaluate the effect of all 4 modules of the training programme.

\section{References}

1. Kabat-Zinn J. Full Catastrophe Living: Using the Wisdom of Your Body and Mind to Face Stress, Pain, and Illness. 15th ed. New York: Delta Trade Paperback/Bantam Dell, 2005.

2. Stanley S. Intimate distances: William James' introspection, Buddhist mindfulness, and experiential inquiry. New Ideas in Psychology 2012;(2):201-211.

3. Baer RA, Smith GT, Lykins E, et al. Construct validity of the five facet mindfulness questionnaire in meditating and non-meditating samples. Assessment 2008;15(3):329-342.

and non-meditating samples. Assessment 2008;15(3):329-342.
4. Baer RA. Mindfulness training as a clinical intervention: A conceptual and empirical review. Clinical Psychology: Baer RA. Mindfulness training as a clinical
Science and Practice 2003;10(2):125-143.

5. Cullen M. Mindfulness-based interventions: An emerging phenomenon. Mindfulness 2011;2:186-193.

6. Hölzel BK, Carmody J, Vangel M, et al. Mindfulness practice leads to increases in regional brain gray matter density. Psychiatry Res 2011;191(1): 36-43. [http://dx.doi.org/10.1016/j.pscychresns.2010.08.006]

7. Davidson RJ, Kabat-Zinn J, Schumacher J, et al. Alterations in brain and immune function produced by mindfulness meditation. Psychosom Med 2003;65(4):564-570.

8. Britton WB, Bootzin RR, Cousins JC, Hasler BP, Peck T, Shapiro SL. The contribution of mindfulness practice to multicomponent behavioral sleep intervention following substance abuse treatment in adolescents: A treatmentdevelopment study. Substance Abuse 2010;31(2):86-97.

9. Crane RS, Kuyken W, Hastings RP, Rothwell N, Williams JMG. Training teachers to deliver mindfulness-based interventions: Learning from the UK experience. Mindfulness 2010;1:74-86.

10. Baum C, Kuyken W, Bohus M, Heidenreich T, Michalak J, Steil R. The psychometric properties of the Kentucky Inventory of Mindfulness Skills in clinical populations. Assessment 2010;17:220-229.

11. Sheldon KM, Ryan RM, Reis H. What makes for a good day? Competence and autonomy in the day and in the person. Personality and Social Psychology Bulletin 1996;22:1270-1279.

12. Neff KD. Development and validation of a scale to measure self-compassion. Self and Identity 2003;2:223250

13. Cohen S, Kamarck T, Mermelstein RA. Global measure of perceived stress. J Health Soc Behav 1983;24:385-396

14. Crane RS, Kuyken W, Williams JMG, Hastings RP, Cooper L, Fennel MJV. Competence in teaching mindfulnessbased courses: Concepts, development and assessment. Mindfulness 2012;3(1):76-84. [http://dx.doi.org/10.1007 s12671-011-0073-2]

15. Roth B, Robbins D. Mindfuless-based stress reduction and health-related quality of life: Findings from a bilingual inner city patient population. Psychosom Med 2004;66(1):113-123.

16. Del Canale S, Louis DZ, Maio V, et al. The relationship between physician empathy and disease complications: An empirical study of primary care physicians and their diabetic patients in Parma, Italy. Acad Med 2012;87:12431249 .

17. Rossouw L, Seedat S, Emsley RA, Suliman S, Hagemeister D. The prevalence of burnout and depression in medical doctors working in the Cape Town Metropolitan Municipality community healthcare clinics and district hospitals of the Provincial Government of the Western Cape: A cross-sectional study. S Afr Fam Pract district hospitals of

18. Goodman MJ, Schorling JB. A mindfulnes course decreases burnout and improves well-being among healthcare providers. Int J Psychiatry Med 2012;43:119-128. 